NOTA CIENTÍFICA

\title{
DETERMINAÇÃO DO VALOR ENERGÉTICO POR CALORIMETRIA DIRETA DE ALGUNS ALIMENTOS CONSUMIDOS POR CRIANÇAS E ADOLESCENTES
}

\section{CALORIC VALUE IN FOODS CONSUMED BY CHILDREN AND ADOLESCENTS DETERMINED THROUGH DIRECT CALORIMETRY}

\author{
Andrea Ferreira Schuwartz TANNUS' \\ Roberta Loraine Valença de CARVALHO' \\ Luciana Pinto RODRIGUES \\ Monica Silva de Souza MEIRELLES' \\ Gilberto João PADOVAN' \\ Júlio Sérgio MARCHINI'
}

\section{INTRODUÇÃO}

Obesidade, diabetes mellitus, insuficiência renal e dislipidemias entre inúmeras outras são condições mórbidas nas quais a modificação dietética é parte essencial do tratamento (Allison et al., 1993). Diante disso, a determinação e quantificação dos nutrientes de alimentos torna-se fundamental para a realização de um planejamento alimentar adequado. O planejamento dietético geralmente é realizado com informações contidas nas tabelas de composição de alimentos, como a do Instituto Brasileiro... (1977), a de Franco (1992) e a Tabela Brasileira de Composição de Alimentos (Lajolo et al., 2000), ou nos rótulos de produtos industrializados. As informações nutricionais, entretanto, não devem ser consideradas absolutas, pois os valores energéticos e nutricionais de um mesmo alimento variam consideravelmente entre as citadas fontes de informação. Além disso, diversos produtos freqüentemente consumidos por crianças e adolescentes como balas, doces, biscoitos e bolachas (Sawaya et al., 1995), raramente são encontrados nestas tabelas.

Philippi et al. (1995), analisando diferentes tabelas nacionais e internacionais concluíram que para um mesmo grupo de alimentos, os teores de carboidratos, lipídeos, proteína, vitamina A, vitamina C, fibras e energia diferem, o que certamente influencia no cálculo final de uma dieta, tanto para macro, quanto para micronutrientes. Outros autores (Pedrosa et al., 1994; Burgos et al., 1996), analisando vários alimentos de preparações usuais na dieta brasileira, concluíram que as informações disponíveis nas Tabelas de Composição de Alimentos necessitam ser revistas.

Estas variações podem, em grande parte, ser devido às diferenças metodológicas utilizadas para a determinação dos conteúdos energético e/ou nutricional. O valor energético em particular é freqüentemente obtido pela soma dos teores de proteínas (4 Kcal/g), lipídeos (9 kcal/g) e carboidratos (4 Kcal/g) (Lajolo \& Vannucchi, 1987). A abordagem mais adequada para se obter o valor energético de alimentos é a calorimetria direta, a qual permite a determinação precisa do conteúdo energético, por meio da combustão completa do alimento.

Assim, o objetivo deste trabalho foi determinar o valor energético de um grupo de balas, doces, biscoitos e salgadinhos por meio de calorimetria direta e compará-lo com informações obtidas nas embalagens.

\section{MATERIAL E MÉTODOS}

Foram adquiridas três amostras de cada tipo de alimento industrializado, provenientes de lotes diferentes, em Ribeirão Preto, SP.

A determinação do valor energético foi realizada por meio de calorimetria direta. Para tanto, as amostras foram secas em estufa ventilada à temperatura de

\footnotetext{
(1) Departamento de Clínica Médica, Divisão de Nutrição, Faculdade de Medicina de Ribeirão Preto, Universidade de São Paulo. Av. Bandeirantes, 3600, Monte Alegre, 14049-900, Ribeirão Preto, SP, Brasil. Correspondência para/Correspondence to: A.F.S. TANNUS.
} 
$100-110^{\circ} \mathrm{C}$ até ficarem completamente desidratadas. Em seguida, foram maceradas com pistilo em almofariz e triplicatas de $1 \mathrm{~g}$ de cada amostra foram utilizadas para determinação do valor energético em bomba calorimétrica (1261 Automatic Isoperibol Bomb Calorimeter - Parr Instrument Company).

A análise comparativa dos valores energéticos foi realizada entre os valores obtidos por calorimetria direta e os informados nos rótulos dos produtos. Todas às vezes que o coeficiente de variação encontrado foi maior que $0,5 \%$, a amostra foi repreparada e medida novamente, sempre em triplicata.

\section{RESULTADOS}

Usualmente o valor energético de alimentos é apresentado em calorias. No entanto, recomenda-se que as diferentes formas de energia sejam expressas quantitativamente em joules (J), do Sistema Internacional de Unidades. Entretanto, de maneira análoga ao Projeto Integrado de Composição de Alimentos da Faculdade de Ciências Farmacêuticas da Universidade de São Paulo (Lajolo et al., 2000), optou-se por apresentar os respectivos resultados na forma de Kcal. Também é importante ressaltar, que esta forma de apresentação de resultados é rotineiramente utilizada por profissionais da área de nutrição. Se houver necessidade de conversão, deve-se considerar que uma quilocaloria equivale a 4,1868 quilojoules (KJ), ou $1 \mathrm{KJ}$ é equivalente a 0,2388 kcal.

Quarenta e um por cento dos alimentos apresentaram um percentual de variação, entre o valor energético medido e o estimado menor que $5 \%$. Os demais (59\%) apresentaram uma diferença acima desse percentual. Podemos ressaltar que o valor energético de alguns alimentos encontrava-se subestimado em seus rótulos em mais de $20 \%$, como o biscoito fibra cracker (22\%), o salgadinho sabor bacon (38\%), a bala dura de menta (45\%), o leite achocolatado (52\%) e a bala dura de cereja (67\%) (Anexo).

\section{DISCUSSÃO}

Allison et al. (1993) analisaram, por meio de calorimetria direta, alguns alimentos com divulgação nacional, outros com distribuição regional e ainda alimentos preparados localmente. Os resultados obtidos evidenciaram que os valores energéticos informados nos rótulos dos produtos preparados localmente e dos distribuídos regionalmente encontravam-se subestimados quando comparados com os valores energéticos dos alimentos obtidos por calorimetria direta. Em conformidade com os nossos resultados, estes autores sugerem que os dados existentes nos rótulos dos produtos possam ser uma fonte inadequada de informações.

Assim, para garantir uma maior confiabilidade a um planejamento dietético, as informações contidas nas tabelas e nos rótulos devem apresentar credibilidade. Daí a importância de se utilizar a calorimetria direta para determinação do valor energético dos alimentos, pois esta técnica é menos sujeita a erros. Diante dos resultados obtidos evidencia-se a importância de se conhecer a metodologia utilizada nas tabelas para servir como instrumento de consulta e avaliação para profissionais de saúde.

\section{AGRADECIMENTOS}

Este trabalho foi parcialmente financiado pela Fundap e pela Faepa.

\section{REFERÊNCIAS BIBLIOGRÁFICAS}

ALLISON, D.B., HESHKA, S., SEPULVEDA, D., HEYMSFIELD, S.B. Couting Calories - Caveat Emptor. JAMA, Chicago, v.270, p.1454-1456, 1993.

BURGOS, M.G.P.A., RIBEIRO, M.A., MELO FILHO, S.C., CABRAL, P.C. Composição centesimal de ovo de galinha, carne de boi e de frango consumidos na área metropolitana do Recife, Nordeste Brasileiro. Revista de Nutrição da Puccamp, Campinas, v.9, n.2, p.224-235, 1996.

FRANCO, G.V.E. Tabela de composição química de alimentos. 6.ed. São Paulo : Atheneu, 1992. p.8-10.

INSTITUTO BRASILEIRO DE GEOGRAFIA ESTATÍSTICA (IBGE). Estudo Nacional da Despesa Familiar - (ENDEF). Tabela de composição de alimentos. Rio de Janeiro, 1977. (Publicações especiais, v.3).

LAJOLO, F.M., VANNUCCHI, H. Tabelas de composição de nutrientes em alimentos: situação no Brasil e necessidades. Archivos Latinoamericanos de Nutrición, Guatemala, v.37, n.4, p. 52-63, 702713, 1987.

LAJOLO, F.M, MENEZES, E.W., PENTEADO, M.D.V.C, FILISETII, T.M.C.C., MARQUEZ, U.M.L. Tabela brasileira de composição de alimentos. Projeto integrado de composição de alimentos. Faculdade de Ciências Farmacêuticas da Universidade de São Paulo. Disponível em: < http:/ /www.fcf.usp.br/tabela/Danecred/index.htm>. Acesso em: 2000.

SAWAYA, A.L., DALLAL, G., SOLYMOS, G. et al. Obesity and malnutrition in a shantytown population in the city of São Paulo, Brazil. Obesity Research, Baton Rouge, v.3, n.2, p.107s-115s, 1995.

PEDROSA, L.F.C., ARAÚJO, M.O.D., LIMA, E.B., MELO, M.S.O.N., GODEIRO, L.M.T. Análise química de preparações usuais em cardápios populares brasileiros. Revista de Nutrição da Puccamp, Campinas, v.7, n.1, p.48-61, 1994.

PHILIPPI, S.T., RIGO, N., LORENZANO, C. Estudo comparativo entre tabelas de composição química de alimentos para avaliação de dietas. Revista de Nutrição da Puccamp, Campinas, v.8, n.2, p.200-213, 1995.

Recebido para publicação em 3 de janeiro de 2000 e aceito 22 de março de 2001. 
ANEXO

VALOR ENERGÉTICO MEDIDO E ESTIMADO DE ALGUNS ALIMENTOS CONSUMIDOS PRINCIPALMENTE POR CRIANÇAS E ADOLESCENTES

\begin{tabular}{|c|c|c|c|}
\hline \multirow{2}{*}{ Alimento } & \multicolumn{2}{|c|}{ Valor energético } & \multirow{2}{*}{ Diferençat } \\
\hline & Medido* (kcal/100 g) & Estimado $\neq(\mathrm{kcal} / 100 \mathrm{~g})$ & \\
\hline Bala dura de cereja e uvab & $42 \pm 0,014(0,003)$ & 382 & 10 \\
\hline Bala dura de laranja e acerolab & $413 \pm 0,154(0,037)$ & 381 & 8 \\
\hline Bala dura de menta ${ }^{b}$ & $401 \pm 0,015(0,004)$ & 383 & 5 \\
\hline Bala dura de cereja ${ }^{b}$ & $395 \pm 0,785(0,296)$ & 236 & 67 \\
\hline Bala dura de menta ${ }^{b}$ & $342 \pm 0,952(0,204)$ & 236 & 45 \\
\hline Bala dura de cereja $^{\mathrm{b}}$ & $396 \pm 0,011(0,003)$ & 383 & 3 \\
\hline Bala dura de eucalipto ${ }^{b}$ & $386 \pm 0,016(0,004)$ & 383 & 1 \\
\hline Bala dura de eucalipto ${ }^{\mathrm{C}}$ & $395 \pm 0,047(0,012)$ & 383 & 3 \\
\hline Bala dura de hortelã ${ }^{b}$ & $394 \pm 0,033(0,008)$ & 383 & 3 \\
\hline Bala dura de limão e hortelã ${ }^{b}$ & $377 \pm 0,046(0,012)$ & 381 & -1 \\
\hline Bala dura de maçã verde $\mathrm{b}^{\mathrm{b}}$ & $396 \pm 0,027(0,007)$ & 383 & 3 \\
\hline Bala dura de morango ${ }^{b}$ & $395 \pm 0,007(0,002)$ & 383 & 3 \\
\hline Bala mastigável de bananab & $393 \pm 0,006(0,002)$ & 381 & 3 \\
\hline Bala mastigável de goiaba ${ }^{b}$ & $395 \pm 0,001(0,000)$ & 381 & 4 \\
\hline Bala mastigável de maçã verde ${ }^{b}$ & $395 \pm 0,006(0,001)$ & 381 & 4 \\
\hline Bala mastigável de marshmallow b & $397 \pm 0,004(0,001)$ & 381 & 4 \\
\hline Bala mastigável de morango ${ }^{\mathrm{a}}$ & $396 \pm 0,003(0,001)$ & 381 & 4 \\
\hline Bala mastigável de morango ${ }^{\text {b }}$ & $396 \pm 0,021(0,005)$ & 381 & 4 \\
\hline Dropes eucalipto $^{\mathrm{b}}$ & $402 \pm 0,005(0,001)$ & 383 & 5 \\
\hline Dropes eucalipto extra-forte ${ }^{b}$ & $411 \pm 0,008(0,002)$ & 383 & 7 \\
\hline Dropes menta $^{b}$ & $408 \pm 0,021(0,005)$ & 383 & 6 \\
\hline Dropes morango $^{\mathrm{b}}$ & $406 \pm 0,012(0,003)$ & 383 & 6 \\
\hline Dropes cereja ${ }^{b}$ & $400 \pm 0,012(0,003)$ & 383 & 4 \\
\hline Dropes maçã verde ${ }^{b}$ & $396 \pm 0,030(0,008)$ & 383 & 3 \\
\hline Dropes morango ${ }^{\mathrm{b}}$ & $399 \pm 0,020(0,005)$ & 383 & 4 \\
\hline Dropes yellow ${ }^{\mathrm{b}}$ & $397 \pm 0,009(0,002)$ & 383 & 4 \\
\hline Biscoito de polvilho ${ }^{a}$ & $461 \pm 0,012(0,003)$ & 436 & 5 \\
\hline Biscoito doce ${ }^{b}$ & $404 \pm 0,115(0,029)$ & 378 & 7 \\
\hline Biscoito doce de chocolate $^{b}$ & $528 \pm 0,203(0,038)$ & 498 & 6 \\
\hline Biscoito doce de coco ${ }^{b}$ & $515 \pm 0,033(0,007)$ & 469 & 10 \\
\hline Biscoito de leite $^{c}$ & $471 \pm 0,025(0,005)$ & 435 & 8 \\
\hline Biscoito recheado de chocolate branco ${ }^{\mathrm{C}}$ & $530 \pm 0,005(0,001)$ & 490 & 8 \\
\hline Biscoito recheado de marshmallow ${ }^{\mathrm{b}}$ & $532 \pm 0,066(0,013)$ & 494 & 8 \\
\hline Biscoito recheado de chocolate ${ }^{b}$ & $518 \pm 0,027(0,005)$ & 483 & 7 \\
\hline Biscoito recheado de chocolate ${ }^{b}$ & $523 \pm 0,012(0,002)$ & 492 & 6 \\
\hline Biscoito recheado de flocos ${ }^{b}$ & $534 \pm 0,103(0,019)$ & 489 & 9 \\
\hline Biscoito recheado de morango ${ }^{b}$ & $520 \pm 0,026(0,005)$ & 486 & 7 \\
\hline Biscoito água e sal ${ }^{\mathrm{C}}$ & $515 \pm 0,061(0,012)$ & 445 & 16 \\
\hline Biscoito doce rosca de $\operatorname{coco}^{\mathrm{b}}$ & $472 \pm 0,068(0,015)$ & 424 & 11 \\
\hline Biscoito fibra cracker $^{\mathrm{c}}$ & $519 \pm 0,147(0,028)$ & 427 & 22 \\
\hline Biscoito Maizena ${ }^{\mathrm{c}}$ & $480 \pm 0,036(0,008)$ & 430 & 12 \\
\hline Biscoito pavesiano $^{c}$ & $467 \pm 0,288(0,062)$ & 420 & 11 \\
\hline Biscoito recheado com doce de leite ${ }^{b}$ & $557 \pm 0,018(0,002)$ & 498 & 12 \\
\hline Biscoito recheado de morango ${ }^{b}$ & $561 \pm 0,028(0,005)$ & 494 & 13 \\
\hline Biscoito sabor coco ${ }^{b}$ & $504 \pm 0,041(0,008)$ & 434 & 16 \\
\hline Biscoito cream cracker ${ }^{\mathrm{C}}$ & $475 \pm 0,020(0,004)$ & 466 & 2 \\
\hline Biscoito doce tipo de mel ${ }^{b}$ & $396 \pm 0,145(0,037)$ & 382 & 4 \\
\hline Biscoito doce tipo pão de mel com cobertura de chocolate ${ }^{b}$ & $495 \pm 0,150(0,030)$ & 476 & 4 \\
\hline Biscoito maria $^{d}$ & $455 \pm 0,013(0,003)$ & 462 & -2 \\
\hline Wafer sabor doce de leite ${ }^{c}$ & $579 \pm 0,077(0,013)$ & 557 & 4 \\
\hline Wafer sabor chocolate ${ }^{c}$ & $583 \pm 0,095(0,016)$ & 550 & 6 \\
\hline Wafer sabor morango $^{\mathrm{c}}$ & $582 \pm 0,501(0,086)$ & 545 & 7 \\
\hline Salgadinho sabor queijo $^{c}$ & $442 \pm 0,611(0,138)$ & 412 & 7 \\
\hline Salgadinho sabor bacon ${ }^{c}$ & $557 \pm 0,148(0,027)$ & 404 & 38 \\
\hline Salgadinho sabor natural ${ }^{\mathrm{C}}$ & $446 \pm 0,036(0,008)$ & 400 & 12 \\
\hline Salgadinho sabor presunto $^{\mathrm{C}}$ & $454 \pm 0,131(0,029)$ & 410 & 11 \\
\hline Salgadinho sabor queijo ${ }^{\mathrm{C}}$ & $483 \pm 0,097(0,020)$ & 430 & 12 \\
\hline Salgadinho sabor pizza ${ }^{\mathrm{c}}$ & $446 \pm 0,422(0,095)$ & 429 & 4 \\
\hline Pão de mel branco ${ }^{b}$ & $391 \pm 0,035(0,009)$ & 378 & 3 \\
\hline Pão de mel com chocolate ${ }^{b}$ & $479 \pm 0,059(0,012)$ & 476 & 1 \\
\hline Leite achocolatado $^{\mathrm{a}}$ & $825 \pm 0,863(0,105)$ & 393 & 52 \\
\hline
\end{tabular}

$\mathrm{a}=$ fabricante $1 ; \mathrm{b}=$ fabricante $2 ; \mathrm{c}=$ fabricante $3 ; \mathrm{d}=$ fabricante 4 .

* Valor energético medido, média \pm Desvio-padrão (coeficiente de variação).

₹ Valor energético estimado pelo rótulo.

+ Diferença percentual entre o valor energético medido e o estimado. 